\title{
On the alignment of Classical T Tauri stars with the magnetic field in the Taurus-Auriga molecular cloud
}

\author{
F. Ménard ${ }^{1}$ and G. Duchêne $e^{1,2}$ \\ ${ }^{1}$ Laboratoire d'Astrophysique, Observatoire de Grenoble, CNRS/UJF UMR 5571, 414 rue de la Piscine, BP 53, \\ 38041 Grenoble Cedex 9, France \\ e-mail: menard@obs.ujf-grenoble.fr \\ 2 Department of Physics and Astronomy, UCLA, Los Angeles, CA 90095-1562, USA \\ e-mail: duchene@astro.ucla.edu
}

Received 24 May 2004 / Accepted 17 June 2004

\begin{abstract}
In this paper we readdress the issue of the alignment of Classical T Tauri stars (CTTS) with the magnetic field in the Taurus-Auriga molecular cloud. Previous studies have claimed that the jet axis of active young stellar objects (YSO), projected in the plane of the sky, is aligned preferentially along the projected direction of the local magnetic field. We re-examine this issue in view of the numerous high angular resolution images of circumstellar disks and micro-jets now available. The images show that $\mathrm{T}$ Tauri stars as a group are oriented randomly with respect to the local magnetic field, contrary to previous claims. This indicates that the magnetic field may play a lesser role in the final stages of collapse of an individual prestellar core than previously envisioned. The current database also suggests that a subsample of CTTS with resolved disks but without observations of bright and extended outflows have a tendency to align perpendicularly to the magnetic field. We discuss the possibility that this may trace a less favorable topology, e.g., quadrupolar, for the magnetic field in the inner disk, resulting in a weaker collimated outflow.
\end{abstract}

Key words. stars: formation - stars: magnetic fields - stars: winds, outflows - stars: planetary systems: protoplanetary disks stars: pre-main sequence

\section{Introduction}

The Taurus molecular cloud complex is one of the best studied star-forming regions. However, it is not typical of most other such regions in that it does not contain massive stars. This is an advantage for studying low-mass star formation because these massive stars have a direct and large impact on their environment, complicating the identification of the processes responsible for the more quiescent solar-like star formation. Also important, Taurus is located nearby and suffers only low extinction. As a consequence, its stellar population is well determined and the census essentially complete. The molecular gas distribution is known to be filamentary (e.g., Schneider \& Elmegreen 1979; Scalo 1990; Mizuno et al. 1995), and oriented mostly perpendicular to the projected large scale magnetic field. These observations provided early support for the models because, due to the properties of magnetic forces, collapse is expected to be easier along magnetic field lines. Except for a few isolated groups, the young stellar population of the Taurus cloud also forms filaments, or "bands" distributed parallel to the molecular filaments (Hartmann 2002).

On a smaller scale, Galli \& Shu (1993) showed that the collapse of an isothermal sphere threaded by straight magnetic field lines results in a pseudo-disk oriented perpendicular to the direction of the original magnetic field. If a centrifugally supported disk forms along the same direction then one expects, in this simple picture of quasi-static, magnetically-driven, isolated star formation, that young stars should have their disks oriented with their major axis perpendicular to and their jet oriented parallel to the local magnetic field. Early observations provided support for this view. Strom et al. (1986) noted during a deep imaging survey of YSO driving HH objects in Taurus that jets and outflows have a tendency to align with the local magnetic field. Similarly, Tamura \& Sato (1989) later argued that disks were preferentially perpendicular to the local magnetic field based on a correlation between the linear polarisation and the magnetic field position angles (PAs).

However, the complete picture of solar-like star formation may prove once again more complicated than expected. Goodman et al. (1990) showed that, although the magnetic field may control the large scale direction of the collapse of molecular clouds in Taurus and Perseus, it does not do so with an "iron grip". If, at large scales, the cloud structure indeed appears filamentary, elongated perpendicular to the magnetic field, significant deviations are found between the direction of the magnetic field and the shape of molecular clouds on 
smaller scales. Collapsing cores are expected to be elongated and the observed average aspect ratio of 2.4 found in the optical by Lee $\&$ Myers (1999) is comparable with the predictions. Interestingly, the cores also show a tendency to be elongated along the filaments (Myers et al. 1991). However, for statistical reasons, these cores are more likely prolate than oblate as they would otherwise all need to be almost perfectly edge-on (Ryden 1996; Curry 2002). This is in apparent contradiction with the above picture for isolated and quasi-static star formation (e.g., Hartmann 2002). In particular, it is unclear how prestellar cores (or filaments) that are elongated perpendicular to the magnetic field can later produce CTTS with jets now aligned with the magnetic field.

Here we investigate the influence of the magnetic field on the process of star formation by re-addressing the issue of the orientation of CTTS with respect to the magnetic field. We use recent high-angular resolution images of T Tauri stars located in Taurus to find the orientation of the symmetry axis of each stellar system and compare it to the local magnetic field. In Sect. 2 we describe the data sets for the stars and the orientation of the magnetic field in Taurus. In Sect. 3 we present the results, i.e., their relative orientations. In Sect. 4 we show that CTTS are oriented randomly with respect to the magnetic field, the implication of which we discuss in Sect. 5. In Sect. 6 we discuss the possible impact of the specific orientation of a source on the properties of the jet it drives. We summarize our findings in Sect. 7.

\section{The data}

\subsection{Orientation of $T$ Tauri stars}

\subsubsection{The database}

In Table 1, we have compiled a catalogue of 37 classical T Tauri stars (CTTS) in the Taurus molecular cloud complex for which a spatially resolved jet and/or disk has been observed. We restrict our study to the zone $4 \mathrm{~h} 00<\alpha<5$ h00 in right ascension and $+17^{\circ}<\delta<+31^{\circ}$ in declination. The final sample contains twenty six (26) objects in which a jet or outflow is detected and resolved well enough to estimate its PA. Most of them, fifteen (15), also have a resolved disk. Additionaly, eleven (11) objects are tabulated where only a disk is resolved, but without signs of extended outflow. In this sample we considered HH 30 (Burrows et al. 1996) and IRAS 04158+2805 (Ménard et al. 2004) as normal CTTS, based on spectroscopic evidence. Their peculiar photometric properties come from their highly inclined orientation.

Whenever possible we have defined the symmetry axis of a CTTS by the orientation of its jet. Otherwise, we have assumed that the symmetry axis of the CTTS lies in the direction perpendicular to the major axis of its disk. In all cases where both a jet and disk have been resolved but two (FT Tau, and DO Tau, but see Sect. 2.1.2), the PAs of both structures are perpendicular to one another to $\sim 15^{\circ}$ or better, as one predicts for example with magneto-hydrodynamical (MHD) wind models. Since the estimates of the jet and disk orientations are independent, this result supports the reliability of the PAs presented in Table 1.
When available, we have quoted published error bars for the orientations. The following estimates may be used as guidelines. Collimated jets are usually identified in deep narrowband images (e.g., Mundt et al. 1991; Dougados et al. 2000) or through long-slit (e.g., Hirth et al. 1997) or slitless (Hartigan et al. 2004) spectroscopic observations. In most cases, jets are clearly resolved and their PAs are known within $\pm 10^{\circ}$ or better.

Disks around young stars can be identified in two main ways: thermal imaging in the submillimeter and millimeter ranges and scattered light imaging in the optical and nearinfrared. For practical reasons, the latter technique is usually limited to edge-on disks (e.g., Burrows et al. 1996; Stapelfeldt et al. 1998) and to circumbinary disks (e.g., Roddier et al. 1996; Close et al. 1998). For the former, a dark lane is usually well defined and the PAs are known very well, usually $\pm 1^{\circ}$. This is the case for HH 30, HV Tau C, and HK Tau B for example.

At radio wavelengths, dust continuum images of disks are obtained with long baseline interferometers (e.g., Dutrey et al. 1996; Kitamura et al. 2002). Furthermore, molecular-line images of disks reveal clear velocity gradients that are consistent with Keplerian rotation (e.g., Simon et al. 2000). When available, we used the resolved CO maps to define the orientation of the disk's semi-major axis. Disk orientations are known to $\pm 5^{\circ}$ or so when a Keplerian velocity gradient is detected and to $\pm 5-15^{\circ}$ otherwise, depending on the inclination.

\subsubsection{Notes on individual objects}

DD Tau. Gomez de Castro \& Pudritz (1992) observed a double-peaked structure for DD Tau which they interpreted as "focal points" of a jet above and below the disk plane. However, Leinert et al. (1993) found DD Tau to be a binary system with the same separation and PA. Furthermore, Hartigan \& Kenyon (2003) obtained visible spectra of each component. They both show a continuum, with an M 3.5 spectral type, rejecting the possibility that these peaks are stationary shocks in a jet. On the other hand, Hartigan et al. (2004) obtained slitless spectroscopy images of the system, revealing a jet emanating from the primary at PA $\sim 125^{\circ}$, along which Gomez de Castro \& Pudritz (1992) had indeed observed spatially-resolved low level forbidden line emission. We adopt this PA for the jet of DD Tau A in this study.

FT Tau. Stapelfeldt et al. (2004, in prep.) suggest the presence of a small $\mathrm{HH}$-like nebulosity at $1^{\prime \prime} 0$ and PA $=209^{\circ}$ from FT Tau. This is the value quoted in Table 1 for the jet PA, once transformed to the range $[0,180[$. The feature is at the detection limit of their HST/WFPC2 image, and remains unconfirmed. The PA given by Dutrey et al. (1996), $82^{\circ} \pm 17^{\circ}$, for the dust continuum emission detected at $2.7 \mathrm{~mm}$, i.e., the PA of the disk, relies on data obtained with a low resolution configuration at the IRAM interferometer. The PA of the jet is better defined and we use this value in the comparison with the magnetic field.

UZ Tau E. The CO disk suggested by Jensen et al. (1996) is aligned very closely with the optical jet detected by 
Table 1. Orientation of T Tauri stars in Taurus-Auriga.

\begin{tabular}{|c|c|c|c|c|c|c|c|c|c|}
\hline Object & $\alpha(2000)$ & $\delta(2000)$ & $\begin{array}{c}\text { PA (jet) } \\
\circ\end{array}$ & Ref. & $\begin{array}{c}\text { PA (disk) } \\
\circ\end{array}$ & Ref. & $\begin{array}{c}\text { PA }(\boldsymbol{B}) \\
\circ\end{array}$ & $\rho_{\mathrm{IS}} / N_{\mathrm{IS}}$ & $\begin{array}{c}|\Delta \mathrm{PA}| \\
\circ\end{array}$ \\
\hline (1) & (2) & (3) & (4) & (5) & (6) & (7) & (8) & (9) & (10) \\
\hline CW Tau & $04^{\mathrm{h}} 14^{\mathrm{m}} 16^{\mathrm{s}} .95$ & $28^{\circ} 10^{\prime} 59^{\prime} 3$ & $144 \pm 2$ & 1 & - & - & 15 & $1.5 / 12$ & 51 \\
\hline DD Tau A & 041831.13 & 281630.1 & 125 & 26 & - & - & 15 & $0.5 / 5$ & 70 \\
\hline CoKu Tau/1 & 041851.54 & 282028.1 & 28 & 11 & 120 & 4 & 15 & $0.5 / 5$ & 13 \\
\hline $04158+2805$ & 041858.2 & 281223 & 165 & 5 & 89 & 5 & 15 & $0.5 / 5$ & 30 \\
\hline RY Tau & 042157.34 & 282636.9 & 110 & 14 & $31 \pm 6$ & 6 & 18 & $0.5 / 10$ & 88 \\
\hline T Tau N & 042159.39 & 193206.8 & 90 & 18 & 19 & 19 & 85 & $1.5 / 4$ & 5 \\
\hline T Tau S & 042159.39 & 193206.1 & 170 & 18 & - & - & 85 & $1.5 / 4$ & 85 \\
\hline Haro 6-5B & 042200.89 & 265737.6 & 53 & 7 & $152 \pm 2$ & 6 & 22 & $0.5 / 32$ & 31 \\
\hline FT Tau & 042339.17 & 245615.1 & 29 & 14 & $82 \pm 17$ & 8 & 73 & $0.5 / 18$ & 44 \\
\hline DG Tau B & 042702.56 & 260530.7 & $111 \pm 8$ & 11 & 32 & 4 & 32 & $0.5 / 16$ & 79 \\
\hline DF Tau & 042702.80 & 254222.3 & 127 & 26 & - & - & 35 & $1.0 / 44$ & 88 \\
\hline DG Tau & 042704.67 & 260616.9 & 42 & 9 & 136 & 10 & 30 & $0.5 / 18$ & 12 \\
\hline Haro 6-10 & 042923.65 & 243302.0 & 60 & 12 & - & - & 74 & $0.5 / 12$ & 14 \\
\hline HН 30 & 043137.6 & 181226 & 31 & 23 & 121 & 23 & 77 & $0.5 / 13$ & 46 \\
\hline HL Tau & 043138.44 & 181358.9 & 51 & 7 & $125 \pm 10$ & 20 & 77 & $0.5 / 13$ & 26 \\
\hline XZ Tau & 043140.02 & 181357.8 & 15 & 21 & - & - & 77 & $0.5 / 13$ & 62 \\
\hline Haro 6-13 & 043215.61 & 242902.3 & 65 & 25 & - & - & 66 & $0.5 / 16$ & 1 \\
\hline UZ Tau E & 043242.93 & 255232.1 & 0 & 12 & $86 \pm 2$ & 2 & 30 & $1.0 / 16$ & 30 \\
\hline GK Tau & 043334.47 & 242107.6 & 20 & 24 & - & - & 50 & $0.5 / 19$ & 30 \\
\hline DL Tau & 043339.04 & 252038.9 & 145 & 14 & $50 \pm 3$ & 2 & 53 & $0.5 / 4$ & 88 \\
\hline HN Tau & 043339.32 & 175152.8 & 170 & 12 & - & - & 77 & $1.0 / 13$ & 87 \\
\hline DO Tau & 043828.57 & 261050.5 & $70 \pm 10$ & 12 & $61 \pm 5$ & 6 & 56 & $0.5 / 7$ & 14 \\
\hline HV Tau C & 043835.29 & 261040.0 & 24 & 14 & 110 & 15 & 56 & $0.5 / 7$ & 32 \\
\hline Haro 6-33 & 044138.9 & 255626 & 60 & 14 & - & - & 52 & $0.5 / 9$ & 8 \\
\hline DP Tau & 044237.66 & 251538.1 & 40 & 16 & - & - & 39 & $0.5 / 5$ & 1 \\
\hline UY Aur & 045147.36 & 304714.1 & 40 & 12 & $135 \pm 5$ & 17 & 65 & $2.0 / 8$ & 25 \\
\hline CY Tau & 041733.73 & 282047.8 & - & - & $150 \pm 7$ & 2 & 15 & $1.0 / 8$ & 45 \\
\hline BP Tau & 041915.82 & 290627.9 & - & - & $152 \pm 3$ & 2 & 175 & $0.5 / 5$ & 67 \\
\hline IQ Tau & 042951.50 & 260646.5 & - & - & $29 \pm 2$ & 6 & 26 & $0.5 / 12$ & 87 \\
\hline HK Tau B & 043150.55 & 242418.4 & - & - & 40 & 13 & 70 & $0.5 / 14$ & 60 \\
\hline GG Tau & 043230.27 & 173141.5 & - & - & $97 \pm 2$ & 22 & 77 & $1.0 / 13$ & 70 \\
\hline DM Tau & 043348.70 & 181010.7 & - & - & $153 \pm 1$ & 2 & 75 & $0.5 / 8$ & 12 \\
\hline CI Tau & 043351.99 & 225030.6 & - & - & $40 \pm 18$ & 8 & 54 & $1.0 / 4$ & 76 \\
\hline AA Tau & 043455.40 & 242853.8 & - & - & $75 \pm 16$ & 6 & 51 & $0.5 / 21$ & 65 \\
\hline DN Tau & 043527.35 & 241459.8 & - & - & $120 \pm 5$ & 6 & 52 & $0.5 / 22$ & 22 \\
\hline LkCa 15 & 043917.80 & 222104.5 & - & - & $61 \pm 1$ & 2 & 54 & $1.5 / 4$ & 83 \\
\hline GM Aur & 045510.95 & 302201.0 & - & - & $51 \pm 2$ & 2 & 65 & $2.0 / 9$ & 76 \\
\hline
\end{tabular}

References: 1) Dougados et al. (2000), 2) Simon et al. (2000), 3) Gomez de Castro \& Pudritz (1992), 4) Padgett et al. (1999), 5) Ménard et al. (2004), 6) Kitamura et al. (2002), 7) Mundt et al. (1991), 8) Dutrey et al. (1996), 9) Lavalley et al. (1997), 10) Kitamura et al. (1996), 11) Eislöffel \& Mundt (1998), 12) Hirth et al. (1997), 13) Stapelfeldt et al. (1998), 14) Stapelfeldt et al. (2004, in prep.), 15) Monin \& Bouvier (2000), 16) Mundt \& Eislöffel (1998), 17) Close et al. (1998), 18) Solf \& Böhm (1999), 19) Akeson et al. (1998), 20) Wilner et al. (1996), 21) Mundt et al. (1990), 22) Guilloteau et al. (1999), 23) Burrows et al. (1996), 24) Aspin \& Reipurth (2000), 25) Strom et al. (1986), 26) Hartigan et al. (2004).

Hirth et al. (1997) and roughly perpendicular to the Keplerian structure clearly identified by Simon et al. (2000). Since the latter observations have a higher spatial resolution and better sensitivity, we assume that the structure discovered by
Jensen et al. corresponds to an outflow instead of a disk. As a confirmation, the optical jet has recently been imaged by Hartigan et al. (2004). 
DO Tau. The extended millimeter-wave structure around DO Tau identified by Kitamura et al. (2002) is oriented along the direction of the optical jet identified by Hirth et al. (1997). It is likely that the emission they detect is in fact related to the jet (e.g., free-free emission) rather than being a disk-like structure. In the following, we consider only the orientation estimated from the optical jet.

Other sources. Finally, we note that the disks surrounding UZ Tau E, UY Aur and GG Tau are not circumstellar disks, but circumbinary disks or rings. The jets associated with the first two systems are likely to come from one of the two stars and it is unclear whether the orientations of the circumbinary disks in these systems are related to the inner binary orientation. However, for both UZ Tau E and UY Aur, the detected jets appear to be perpendicular to the circumbinary disks. For lack of better information regarding the inner circumstellar disks in these binary systems, we assume that they are coplanar and keep the PAs of the circumbinary disks in the analysis below.

\subsection{Direction of the magnetic field in Taurus}

We use published linear polarisation measurements of background stars to trace the direction of the magnetic field projected in the plane of the sky in Taurus. We compiled the polarisation measurements published by Vrba et al. (1976), Heyer et al. (1987), Moneti et al. (1984), Tamura et al. (1987), Tamura \& Sato (1989), and Goodman et al. $(1990,1992)$. These studies cover most of the area we are interested in. To complete the polarisation data set in the vicinity of T Tau itself, we used the polarisation vectors shown in Fig. 1c of Tamura \& Sato (1989). Similarly, in the areas located at $\alpha_{2000}=04 \mathrm{~h} 05 \mathrm{~m}$ and $\delta_{2000}=+26^{\circ}$ and $04 \mathrm{~h} 15 \mathrm{~m} \leq \alpha_{2000} \leq 04 \mathrm{~h} 25 \mathrm{~m}$ and $+28^{\circ} \leq$ $\delta_{2000} \leq+30^{\circ}$, we estimated the local polarisation from Fig. 2 of Tamura \& Sato (1989). Overall, we compiled $\sim 400$ polarisation measurements. Only measurements with $P / \sigma_{\mathrm{P}}>3$ were retained to ensure a good determination of the PA, and hence of the projected direction of the magnetic field.

To estimate the direction of the magnetic field near each target $\mathrm{T}$ Tauri star, we searched the interstellar polarisation database in concentric circles starting with radii of 0.5 , and increasing to $1.0,1.5$, and 2.0 . We selected the smallest radius containing at least 4 different measurements. The median of the PAs in this circle is used as the direction of the local magnetic field close to each T Tauri stars.

UY Aur and GM Aur are located outside the zone containing the bulk of polarisation measurements. In these two cases, the direction of the magnetic field is extrapolated from the nearest measurements, as opposed to be medianed in an area centered on the sources. Within $2^{\circ}$ or so of both sources the magnetic field is well ordered and the extrapolation should be reliable.

\section{Results}

In Table 1 we present the PAs we compiled for CTTS. All angles are measured East of North and are given in the range $\left[0^{\circ}, 180^{\circ}\right.$. Columns 1,2 , and 3 contain the object name and coordinates, respectively. The PA of the jet, PA (jet), and the reference are given in Cols. 4 and 5. The PA of the major axis of the disk, PA (disk), and the reference are in Cols. 6 , and 7. Column 8 is the estimation of the projected direction of the magnetic field, PA $(\boldsymbol{B})$, in the vicinity of the source. For the interstellar polarisation measurements, $\rho_{\text {IS }}$ and $N_{\text {IS }}$ are the radius within which the orientation of the magnetic field is estimated and the number of measurements considered, respectively. They are shown in Col. 9. Finally, the last column contains $|\triangle \mathrm{PA}|$, the difference in PA between the local magnetic field and the symmetry axis of the CTTS. This is the quantity we are interested in.

The results are plotted in Fig. 1. The short, thin vectors trace the projected direction of the magnetic field across the cloud as measured by linear polarisation of background stars. The size of the vectors is uniform and not to scale with the level of linear polarisation. The large scale magnetic field runs roughly in the NE-SW direction (e.g., Tamura \& Sato 1989), with significant bending on smaller scales.

The long, thick vectors trace the projected orientation of each CTTS. Thick vectors with arrow-heads are for sources with a resolved jet/outflow and trace the direction of the flow. Thick vectors without arrow-heads are for sources where only a disk is known today, they trace the direction perpendicular to the major-axis of the disk. Therefore, all thick vectors are tracing the same symmetry axis for the CTTS, namely the axis of rotation (i.e., along the jet or perpendicular to the disk, which is similar). It is also important to note that the error bars on the orientation of CTTS, typically $5^{\circ}-10^{\circ}$ are not reponsible for the large spread observed. The wide distribution of PAs with respect to the magnetic field is a property of the CTTS sample.

\section{Orientation of CTTS in Taurus-Auriga}

Throughout the discussion below we will assume that the symmetry axis of a CTTS+disk system is the same as its rotation axis and its jet/outflow axis. This assumption reflects our current observational and theoretical understanding of the geometry of a T Tauri star and its circumstellar environment. In all figures and histograms, only the symmetry axis of the systems is used. In other words, the $90^{\circ}$ difference between the disk major axis and the jet axis has been taken into account in all plots.

In Fig. 2 we present the cumulative distribution histogram (CDH) of the CTTS sample. The straight dotted-line is the $\mathrm{CDH}$ expected for an infinite perfectly random sample. The solid histogram of the complete CTTS sample follows this line closely. Statistically, the null hypothesis from a Kolmogorov test is accepted, i.e., the observed distribution of CTTS can be drawn from a randomly oriented parent distribution, with only $20 \%$ chance to reject the null hypothesis. This result indicates that the group of CTTS we compiled is very likely randomly oriented with respect to the local magnetic field in Taurus.

This new result contradicts previous claims, e.g., by Strom et al. (1986) and Tamura \& Sato (1989), that young stars were aligned mostly parallel to the magnetic field. The origin of that 


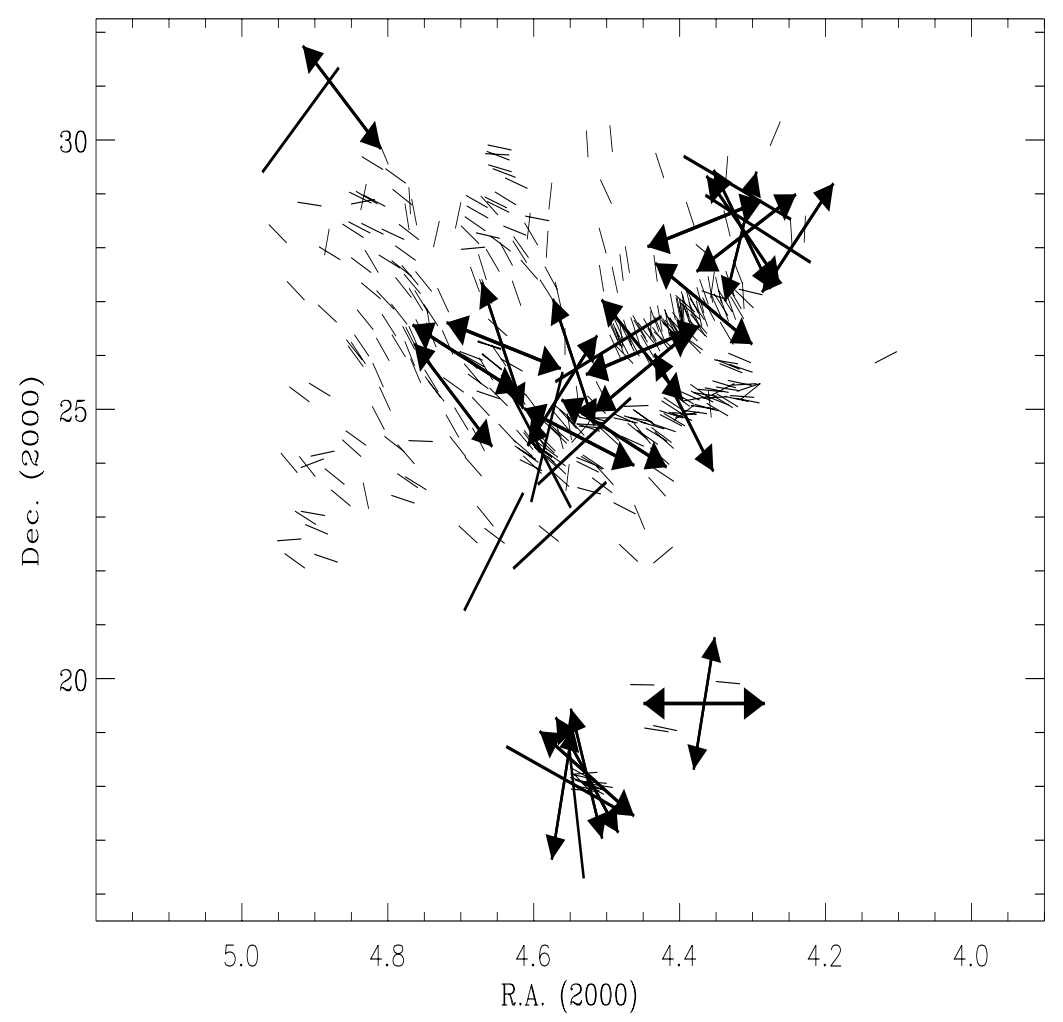

Fig. 1. Plot of the orientation of the stars with respect to the magnetic field. Small segments represent interstellar polarisation measurements while long bold segments indicate the orientation of CTTS. Thick vectors with arrow heads are for CTTS with jets, thick vectors without arrow heads are for CTTS with a disk but no detected extended outflow. Short thin segments trace the direction of the magnetic field.

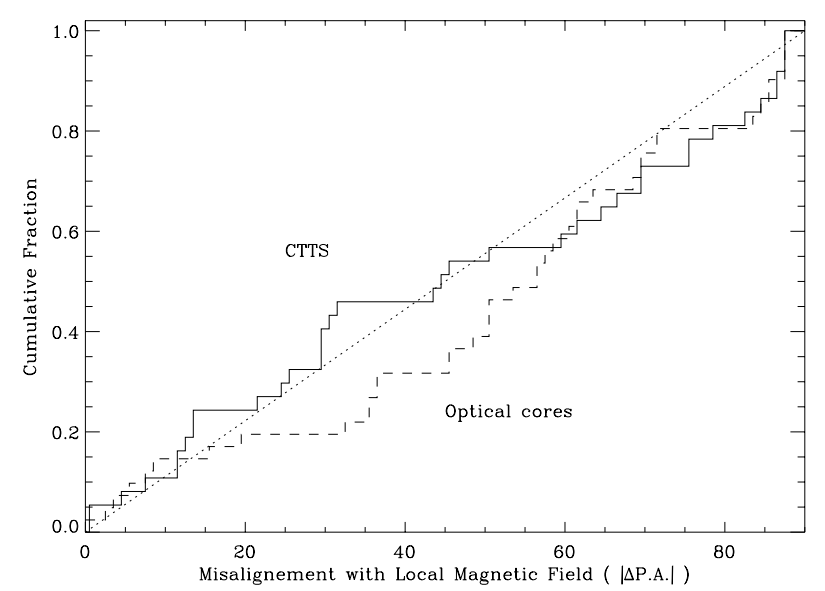

Fig. 2. Cumulative distribution function of the difference in PAs between the local magnetic field and the CTTS symmetry axis (solid histogram, Table 1) and the major axis of optical cores (dashed histogram, from Lee \& Myers 1999). The dotted line is the function expected for an infinite randomly oriented sample.

contradiction lies in the larger size of our sample and in biases in the composition of previous samples.

In the study of Strom et al. (1986) the sample was incomplete and made only of stars with well defined jets or bright Herbig-Haro objects. Many of their sources are included in Table 1; those not included are not CTTS but more embedded YSOs. In the study of Tamura \& Sato (1989), the orientation of the YSOs was deduced from near-infrared polarimetry.
This is a more indirect method that relies on models. Caution must also be used as it furthermore suffers from a $90^{\circ}$ ambiguity, with polarisation vectors being parallel or perpendicular to the disk depending whether or not an envelope is present (e.g., Whitney \& Hartmann 1993). This ambiguity can not be lifted easily without direct imaging. Therefore, the samples used in previous studies were biased towards sources driving bright jets, or with a poorly or ambiguously defined geometry.

On the opposite, the sample in Table 1 is made only of stars where the disks and/or jets are well resolved. The orientation of all sources projected in the plane of the sky is secure and determined without ambiguity. We note that there are selection effects against the detection of both jets and disks in the case of pole-on systems. However, it is unlikely that this bias prevents us from including targets at specific PA with respect to the magnetic field projected on the plane of the sky. Furthermore, our sample contains CTTS only, removing the possible confusion arising from the presence of an extended and dusty envelope around the class I sources contained in other samples.

\section{The influence of magnetic field throughout star formation}

On the large scale, star formation in the Taurus-Auriga molecular cloud appears to be at least partially driven by the magnetic field. First, both the dense gas clouds and the YSOs show a large scale filamentary distribution roughly perpendicular to the magnetic field. Furthermore, at the smaller, individual object scale, Hartmann (2002) showed that pre-stellar cores, 
which are most likely prolate (Curry 2002), are elongated preferentially parallel to the direction of the filaments. These findings contrast with our main result that individual CTTS are randomly oriented with the local magnetic field at a typical age of 1-3 Myr. Are these apparently contradicting results revealing a physical mechanism at play during the late stages of star formation or the mere consequence of poor statistics associated with small samples?

In Fig. 2, we have also plotted the $\mathrm{CDH}$ of the relative angle between the major axis of optical cores from Lee \& Myers (1999) and the local magnetic field in Taurus. We used the orientation of the long axis of the cores as their symmetry axis since they are likely to be prolate (Curry 2002). We have included all cores in the same area of the sky where we have studied CTTS and have used the same method to determine the orientation of the local magnetic field. The CDH of optical cores is systematically under the theoretical distribution for random orientation and the median relative angle is about $55^{\circ}$. As pointed out by Hartmann (2002), the alignment of cores is uneven across Taurus. For example, it is particularly good in a few regions, e.g., his group 3. In general, optical cores have their major axes preferentially oriented perpendicularly to the local magnetic field. As a group, the probability (from a Kolmogorov test) that their distribution can be drawn from a randomly oriented parent distribution is small, only $\sim 8 \%$.

Similarly, the probability that the samples of CTTS and optical cores can be taken from the same randomly oriented parent distribution is only about $11 \%$. In other words, the moderate trend observed for optical cores to be preferentially oriented perpendicular to the local magnetic field does not seem to apply anymore at the later CTTS stage.

The available observations of the Taurus-Auriga molecular cloud and its populations of prestellar cores and CTTS therefore suggest a scenario in which the role of the cloud's magnetic field decreases as star formation proceeds to ever smaller scales. As suggested by Hartmann (2002), the magnetic field has likely driven the early collapse of the entire cloud into several regularly-spaced filaments that are perpendicular to the large scale magnetic field. On the intermediate scale, that of individual cores, this preferred orientation of the cores is still observed, though as a weaker trend. At small scales however, moving towards the formation of individual star+disk systems out of dense cores, the memory of the original direction of collapse appears lost as indicated by the observed random orientation of the CTTS' symmetry axis in the same reference frame of the magnetic field.

This evolution of the distribution of PAs from large to small scales does not necessarily invalidate the current paradigm of isolated, quasi-static star formation. However, it indicates that during the end stages of the star formation process the final orientation of a system may be determined by a stochastic mechanism that becomes independent of the magnetic field.

This decoupling from the early stages could come from the fact that prolate prestellar cores may first evolve towards a quasi-spherical configuration due to their self-gravity (Curry 2002), in line with the observation that protostellar cores are rounder than starless cores (Goodwin et al. 2002). Alternatively

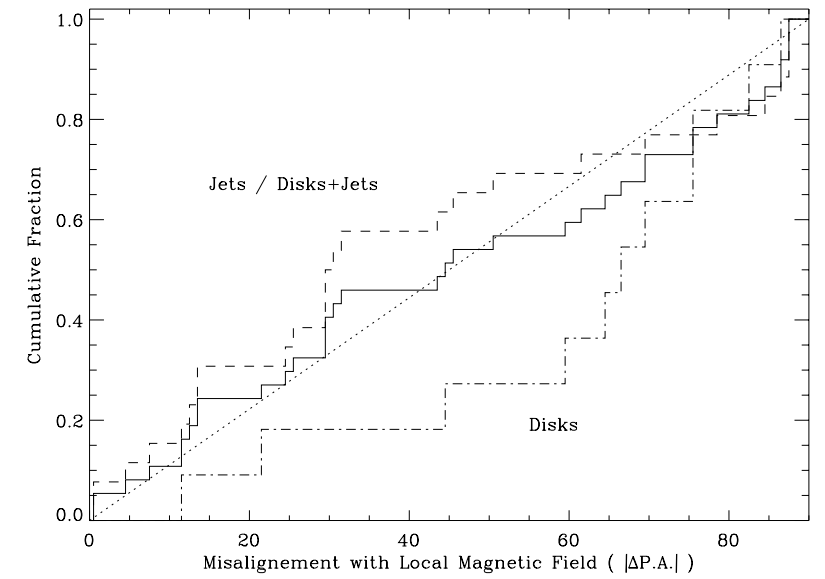

Fig. 3. Cumulative distribution function of the difference in PAs between the local magnetic field and the CTTS symmetry axis (Col. 10 in Table 1). The dashed histogram is for all sources with a jet, the dotdashed histogram for sources with a disk but no jet/outflow and the solid histogram is for the whole sample. The dotted line is the function expected for an infinite randomly oriented sample.

or additionally, these cores could fragment into two or more smaller and more spherical cores, as needed to account for the very high binary frequency among young stars (Hartmann 2002). Because of their proximity, these rounder cores would be more prone to dynamical interactions (for example) that could randomize their orientations.

\section{Exploring the influence of orientation on the jet properties}

In this section we explore the impact of the orientation of a CTTS with respect to the large scale magnetic field on its capacity to launch a powerful and a well collimated outflow. Surprisingly, while a connection between the (random) orientation of a T Tauri stars and the strength of its outflow is a priori not expected in the current models, our results may indicate othwerwise.

Consider all CTTS where only a disk is detected, i.e., the bottom part of Table 1. Surprisingly, they appear to align preferentially at a large angle from the local magnetic field (i.e., with the major-axis of their disk parallel to the magnetic field). This is illustrated in both Figs. 3 and 4. The probability that the $\mathrm{CDH}$ for this subsample is drawn from a randomly distributed sample or from the same parent distribution as the CTTS with a resolved jet are $5 \%$ and $13 \%$, respectively. Due to the small number of sources with only a disk in our sample, these levels are not conclusive. However, they suggest that objects for which a jet has not yet been detected are oriented differently than the complete sample, with a preference to be perpendicular to the local magnetic field.

It is interesting to note however that almost all these sources also have forbidden emission lines which trace mass-loss activity in their spectrum (Cabrit et al. 1990; Hartigan et al. 1995; Hartigan \& Kenyon 2003). This means that mass-loss is most likely present in all the CTTS of Table 1, but it is either not 


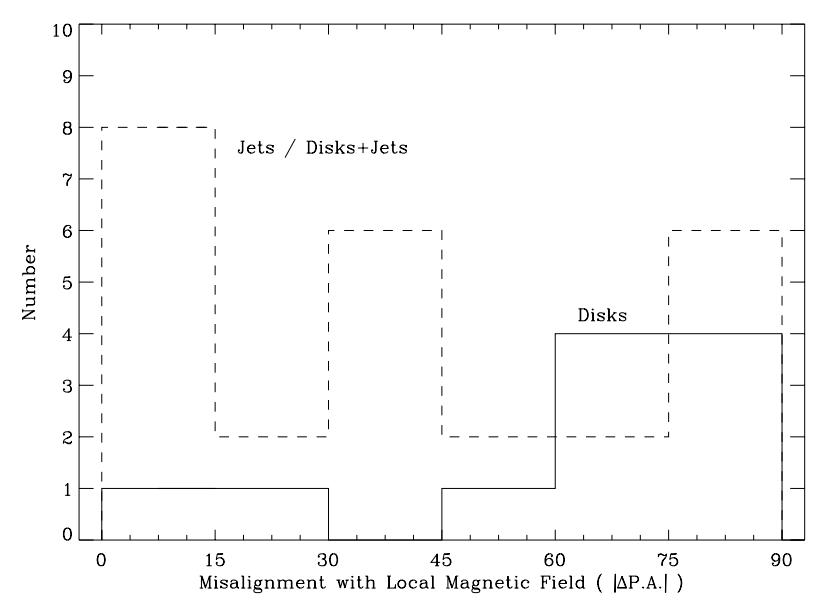

Fig. 4. Histogram of the difference in PAs between the objects' symmetry axes and the local magnetic field. The solid-line histogram represent CTTS with disks but no jet, the dashed-line histogram is for CTTS with a jet.

extended enough or it is too weak to be resolved for the socalled disk-only sources.

Previous claims regarding the orientation of CTTS were based on subsamples made of sources driving bright and well collimated flows. They showed a tendency to align parallel to the magnetic field (e.g., Strom et al. 1986). With improved imaging techniques, weaker and/or shorter jets are now being discovered (e.g., Hartigan et al. 2004; Stapelfeldt et al. 2004, in prep.) Surprisingly, they are found to originate more often from objects whose symmetry axis is oriented far away from the magnetic field. These new jets destroy the correlation previously found.

This lack of correlation is interesting since all CTTS probably still have mass-loss based on the presence of forbidden line emission in their spectrum. As a consequence, the correlation identified in early studies likely does not exist, it is the result of small sample size and selection effects ${ }^{1}$.

An interesting possibility to interpret this result is to suggest that there is a connection between the orientation of a $T$ Tauri star and the strength and/or length of its jet, namely that systems roughly aligned with the local magnetic field are more prone to drive bright, extended and well-defined jets while those largely misaligned cannot develop the proper conditions to drive extensive jet.

This interpretation must still be confirmed with a more quantitative analysis of the jet properties (e.g., surface brightness in specific emission lines, spatial extent, collimation angle, etc.). Yet, our results support such a possibility. If confirmed by future studies, it could indicate that somehow there is a feedback between the configuration of the magnetic field at the stellar surface/inner disk and the direction of the nearby field in the molecular cloud.

1 This is a refinement of our previous suggestion that a better alignment with the magnetic field was found for this sample (Duchêne \& Ménard 2003; Ménard \& Duchêne 2004). At that time, the subsample of sources with spatially resolved jets did not include recent detections of very low surface brightness, poorly collimated outflows.
Current theories of ejection mechanisms for CTTS are based either on the presence of a relatively strong stellar magnetic field (Shu et al. 2000) whose long-range dipolar component interacts with the inner disk or based on the presence of a non-stellar field threading the inner disk (Königl \& Pudritz 2000; Ferreira 1997). The size and topology of the jet-launching region, e.g., more or less extended, depends on the exact model. Noticeably however, Ferreira (1997) showed in the framework of an MHD disk-wind model that a dominant quadrupolar magnetic field configuration leads to a much weaker wind than a dipolar configuration. Our findings therefore support the speculative idea that the magnetic field configuration in the disk and/or magnetosphere of a CTTS may be influenced by its relative orientation with respect to the cloud's magnetic field.

\section{Summary and future work}

We used recent high-angular resolution images of young stars to re-evaluate the importance of the local magnetic field in the late stages of the star formation process. We have focussed on the quiescent Taurus-Auriga star-forming region where massive stars and their strong influence on the cloud dynamics are absent. We have compiled a database of 37 CTTS with spatially resolved collimated jets and/or circumstellar disks. For each object, we have determined the orientation of its symmetry axis on the plane of the sky and compared it to the direction of the local magnetic field.

Whereas previous studies had suggested that young stars form with their symmetry axis parallel to the magnetic field, we find that the population of CTTS in Taurus-Auriga is randomly oriented with respect to the magnetic field. This is the main result of this work. It suggests either that the influence of the cloud's magnetic field on the formation process is dominant at large scales (entire cloud) but largely decreases on the much smaller scale of individual objects or that the orientation has changed since birth.

We also find a speculative connection between the strength of CTTS jets and their orientation with respect to the magnetic field. Bright, elongated, well-collimated jets are preferentially parallel to the magnetic field while weaker, fuzzy, and/or shorter jets tend to be perpendicular to it. This may indicate a link between the orientation of an object with respect to the cloud's magnetic field and the morphology (e.g., dipolar vs. quadrupolar) of the stellar magnetic field it can sustain at long range.

Deeper imaging of CTTS will reveal more jets and disks, allowing us to test this suggestion. In particular, it will be interesting to determine the properties (surface brightness, length, collimation angle) of the jets from those sources for which only a disk has been resolved so far. One can also imagine using submillimeter linear polarisation measurements to study the orientation of much younger embedded protostars. Finally, conducting similar studies in other star-forming regions, such as the much denser $\rho$ Ophiuchus and Orion clouds, will help disentangle the influence of magnetic field on star formation from other physical effects. 
Acknowledgements. The authors acknowledge interesting discussions with Catherine Dougados and Jonathan Ferreira. F.M. also wishes to thank the organisers of the workshop on Solar and Stellar Magnetism held in Toulouse, France, in September 2002 where we had the idea presented in this paper. Financial support from the "Programme National de Physique Stellaire" (PNPS) of CNRS/INSU, France, is gratefully acknowledged. This research has made use of the SIMBAD database, operated at CDS, Strasbourg, France.

\section{References}

Akeson, R. L., Koerner, D. W., \& Jensen, E. L. N. 1998, ApJ, 505, 358 Aspin, C., \& Reipurth, B. 2000, MNRAS, 311, 522

Burrows, C. J., Stapelfeldt, K. R., Watson, A. M., et al. 1996, ApJ, 473,437

Cabrit, S., Edwards, S., Strom, S. E., \& Strom, K. M. 1990, ApJ, 354, 687

Close, L. M., Dutrey, A., Roddier, F., et al. 1998, ApJ, 499, 883

Curry, C. L. 2002, ApJ, 576, 849

Dougados, C., Cabrit, S., Lavalley, C., \& Ménard, F. 2000, A\&A, 356, L41

Duchêne, G., \& Ménard, F. 2003, in Open Issues in Local Star Formation, ed. J. Lépine, \& J. Gregorio-Hetem (Dordrecht: Kluwer), 287

Dutrey, A., Guilloteau, S., Duvert, G., et al. 1996, A\&A, 309, 493

Eislöffel, J., \& Mundt, R. 1998, AJ, 115, 1554

Ferreira, A. 1997, A\&A, 319, 340

Galli, D., \& Shu, F. H. 1993, ApJ, 417, 220

Goodman, A. A., Bastien, P., Ménard, F., \& Myers, P. C. 1990, ApJ, 359,363

Goodman, A. A., Jones, T. J., Lada, E. A., \& Myers, P. C. 1992, ApJ, 399, 108

Goodwin, S. P., Ward-Thompson, D., \& Whitworth, A. P. 2002, MNRAS, 330, 769

Gomez de Castro, A. I., \& Pudritz, R. E. 1992, ApJ, 379, L107

Guilloteau, S., Dutrey, A., \& Simon, M. 1999, A\&A, 348, 570

Hartigan, P., Edwards, S., \& Ghandour, L. 1995, ApJ, 452, 736

Hartigan, P., \& Kenyon, S. J. 2003, ApJ, 583, 334

Hartigan, P., Edwards, S., \& Pierson, R. 2004, ApJ, in press

Hartmann, L. 2002, ApJ, 578, 914

Heyer, M. H., Snell, R. L., Goldsmith, P. F., \& Myers, P. C. 1987, ApJ, 321,855

Hirth, G. A., Mundt, R., \& Solf, J. 1997, A\&AS, 126, 437

Jensen, E. L. N., Koerner, D. W., \& Mathieu, R. D. 1996, AJ, 111, 2431
Kitamura, Y., Kawabe, R., \& Saito, M. 1996, ApJ, 465, L137

Kitamura, Y., Momose, M., Yokogawa, S., et al. 2002, ApJ, 581, 357

Königl, A., \& Pudritz, R. E. 2000, in Protostars and Planets IV, ed. V. Mannings, A. P. Boss, \& S. S. Russell (Tucson: Univ. of Arizona Press), 759

Lavalley, C., Cabrit, S., Dougados, C., Ferruit, P., \& Bacon, R. 1997, A\&A, 327, 671

Lee, C. W., \& Myers, P. C. 1999, ApJS, 123, 233

Leinert, Ch., Zinnecker, H., Weitzel, N., et al. 1993, A\&A, 278, 129

Ménard, F., \& Duchêne, G. 2004, in Magnetic Field and Star Formation: Theory vs. Observation, ed. A. I. Gomez de Castro, $\&$ F. Colomer (Dordrecht: Kluwer Academic), in press

Ménard, F., Dougados, C., Magnier, E., et al. 2004, ApJ, submitted

Mizuno, A., Onishi, T., Yonekura, Y., et al. 1995, ApJ, 445, L161

Moneti, A., Pipher, J. L., Helfer, H. L., McMillan, R. S., \& Perry, M. L. 1984, ApJ, 282, 508

Monin, J.-L., \& Bouvier, J. 2000, A\&A, 356, L75

Mundt, R., \& Eislöffel, J. 1998, AJ, 116, 860

Mundt, R., Bührke, T., Solf, J., Ray, T. P., \& Raga, A. C. 1990, A\&A, 232, 37

Mundt, R., Ray, T. P., \& Raga, A. C. 1991, A\&A, 252, 740

Myers, P. C., Fuller, G. A., Goodman, A. A., \& Benson, P. J. 1991, ApJ, 376, 561

Padgett, D. L., Brandner, W., Stapelfeldt, K. R., et al. 1999, AJ, 117, 1490

Roddier, C., Roddier, F., Northcott, M. J., Graves, J. E., \& Jim, K. 1996, ApJ, 463, 326

Ryden, B. S. 1996, ApJ, 471, 822

Scalo, J. 1990, in Physical Processes in Fragmentation and Star Formation, ed. R. Capuzzo-Dolcetta, C. Chiosi, \& A. DiFazio (Dordrecht: Kluwer), 151

Schneider, S., \& Elmegreen, B. G. 1979, ApJS, 41, 87

Shu, F. H., Najita, J. R., Shang, H., \& Li, Z.-Y. 2000, in Protostars and Planets IV, ed. V. Mannings, A. P. Boss, \& S. S. Russell (Tucson: Univ. of Arizona Press), 789

Simon, M., Dutrey, A., \& Guilloteau, S. 2000, ApJ, 545, 1034

Solf, J., \& Böhm, K.-H. 1999, ApJ, 523, 709

Stapelfeldt, K. R., Krist, J. E., Ménard, F., et al. 1998, ApJ, 502, L65

Strom, K. M., Strom, S. E., Wolff, S. C., Morgan, J., \& Wenz, M. 1986, ApJS, 62, 39

Tamura, M., \& Sato, S. 1989, AJ, 98, 1368

Tamura, M., Nagata, T., Sato, S., \& Tanaka, M. 1987, MNRAS, 224, 413

Vrba, F. J., Strom, S. E., \& Strom, K. M. 1976, AJ, 81, 958

Whitney, B. A., \& Hartmann, L. 1993, ApJ, 402, 605

Wilner, D. J., Ho, P., \& Rodriguez, L. F. 1996, ApJ, 470, L117 